\title{
Inhibition of $\beta$-Amyloid Fibrillization by Directed Evolution of a $\beta$-Sheet Presenting Miniature Protein
}

\author{
Thaddeus J. Smith, Cliff I. Stains, Scott C. Meyer, and Indraneel Ghosh* \\ Contribution from the Department of Chemistry, University of Arizona, Tucson, Arizona 85721 \\ ghosh@email.arizona.edu
}

\section{Supplementary Information}

General Materials and Methods: Enzymes and M13KO7 helper phage were purchased from New England Biolabs, XL1-Blue E. coli was obtained from Stratagene, custom oligonucleotides were obtained from IDT (Integrated DNA Technologies), and all other materials were purchased from Sigma, unless otherwise noted.

\section{Library construction and phage display selection}

The phage display vector pHTB1 was obtained by cloning a synthetic construct encoding for the HTB1 gene between the SfiI and NotI restriction sites into the phagemid vector pCANTAB 5E (Pharmacia). A unique PstI site was incorporated in the HTB1 construct enabling the use of two cassettes for mutagenesis (SfiI/PstI or PstI/NotI). The beta-sheet librarycontaining cassette was created between the two restriction sites SfiI and PstI by incorporating the codon NRK at each randomized position (N corresponds to either A, T, G, or C; R is either A or $\mathrm{G}$, and $\mathrm{K}$ represents $\mathrm{T}$ or $\mathrm{G})$.

Library Forward primer : $5^{\prime}-$ gcggcccagccggccatggcccagaccttcnrkcttnrkatcnrk ggtaaaaccctgnrknrknrkatcnrkatcnrkgctgttgacgctgcagaagctg - 3'

Library Reverse primer: 5' - cagcttctgcagcgtcaacagc - 3'

After transformation of the library into XL-1 Blue E. coli cells, the total library size was $2.2 \times 10^{9}$. The sequence of the completed library was verified by DNA sequencing of isolated single colonies after plating of library-containing E. coli on LB agar plates with ampicillin selection and glucose. A complete description of the HTB1 library is as follows: 


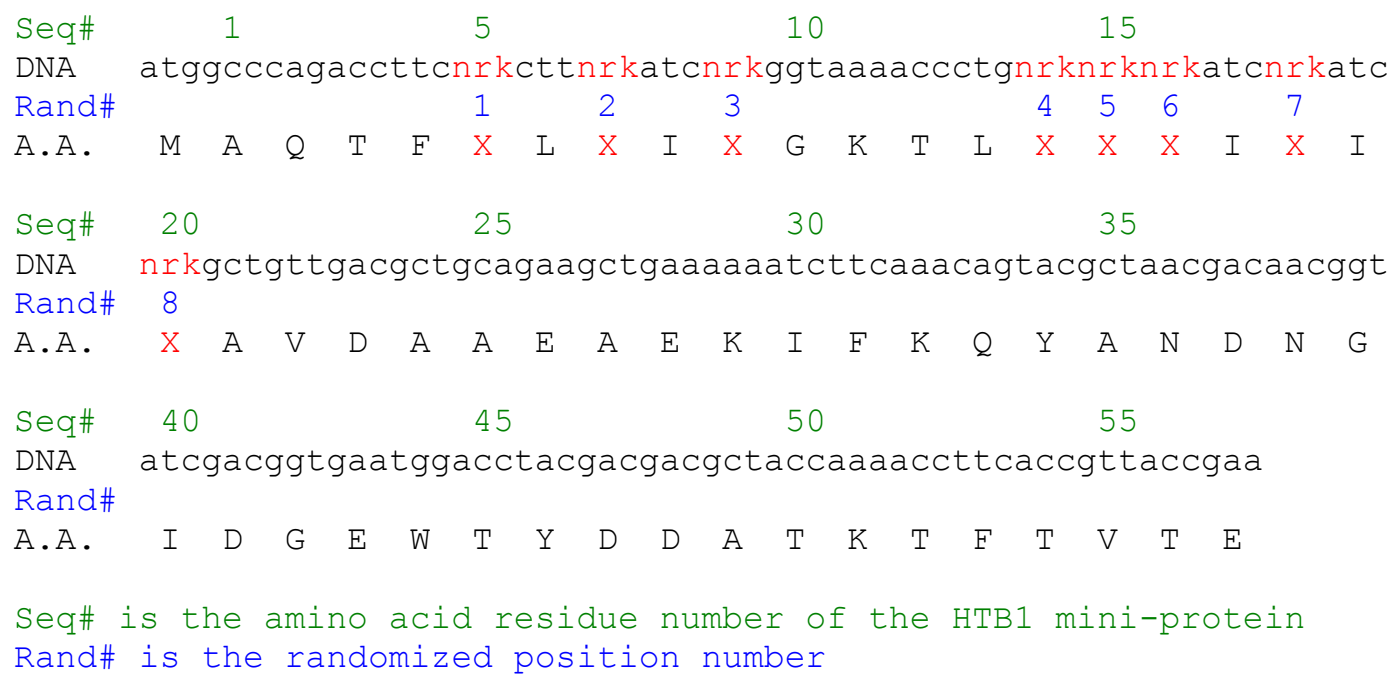

The HTB1 library was displayed as a gene III fusion on M13 phage particles using the library phagemid vector described above. The library-containing phages were isolated from the supernatant (via polyethylene glycol (PEG) precipitation) of an XL-1 Blue E.coli culture after superinfection with M13KO7 helper phage. Targets were immobilized as described in the main text of the manuscript, and selections were carried out using standard methods. Bound phage particles were eluted under acidic high-salt conditions $(0.2 \mathrm{M}$ glycine, $1 \mathrm{M} \mathrm{NaCl}, \mathrm{pH}=2)$. The output phage particles were then used to infect fresh XL-1 Blue E.coli, which became, after amplification, the input for the next round of selection. Phage-infected E. coli were plated on agar plates with glucose and ampicillin selection to achieve single colonies for mutant isolation and DNA sequencing.

One round of panning was carried out against human $\operatorname{IgG}$, which the parent HTB1 is known to bind. " This constitutes a "structural selection" whereby only the mutants that purportedly maintain a fold similar to that of HTB1 can bind to IgG. Next, five rounds of panning were carried out in a "functional selection" against beta-amyloid. In this selection, the goal was to develop a novel function for the HTB1 scaffold, i.e. beta-amyloid binding. The two types of selections thus allow for the isolation of mutants that are both well folded and have novel function, distinct from the parent protein (HTB1).

A handful of mutants were repeatedly observed in the output of the $4^{\text {th }}$ and $5^{\text {th }}$ rounds of selection, but only two mutants were observed in both rounds. The randomized portions of these two selected mutants are listed below (the parent HTB1 is shown for reference):

HTB1: MAQTFKLIINGKTLKGEITIEAVD...

TJ10: MAQTFWLSIQGKTLYWQIRIYAID...

TJ16: MAQTFYLSIQGKTLNENIHINAVD...

TJ10 was chosen for further investigation. This protein also contains a Val to Ile mutation at a position not randomized in the original library. Such spontaneous mutations have been seen previously. ${ }^{2,3}$ 


\section{TJ10 cloning, expression, and purification}

The selected HTB1 mutant, TJ10, was cloned into appropriate restriction sites of the pQE30 His-tag expression vector (Qiagen) using designed primers. The His-tag fusion of TJ10 was expressed in XL-1 Blue E. coli after induction with $1 \mathrm{mM}$ IPTG at an $\mathrm{OD}_{600}=1$. Cells were harvested after 24 hours, spun down and frozen at $-78{ }^{\circ} \mathrm{C}$. The cells were later thawed and thoroughly lysed by sonication. The soluble fraction of the cell lysate was bound to nickel agarose resin (Qiagen) in a buffer containing $50 \mathrm{mM} \mathrm{Na}_{2} \mathrm{HPO}_{4}$ and $0.3 \mathrm{M} \mathrm{NaCl}$ at $\mathrm{pH}$ 8.0. The protein was eluted with increasing concentrations of imidazole, with full elution at $0.5 \mathrm{M}$ imidazole. Subsequent purification was carried out on a size-exclusion HiLoad 16/60 Superdex 75 prep grade FPLC column (Amersham Pharmacia). The typical purified yield of TJ10 was 1 $\mathrm{mg} / \mathrm{L}$ of culture. The molecular weight of TJ10 was confirmed by MALDI mass spectrometry.

Full Sequence of TJ10:

MRGSHHHHHHGSAAHYMAQTFWLS IQGKTLYWQIRIYAIDAAEAEKI FKQYANDNG I DGEWTYDDATKTFTVTE

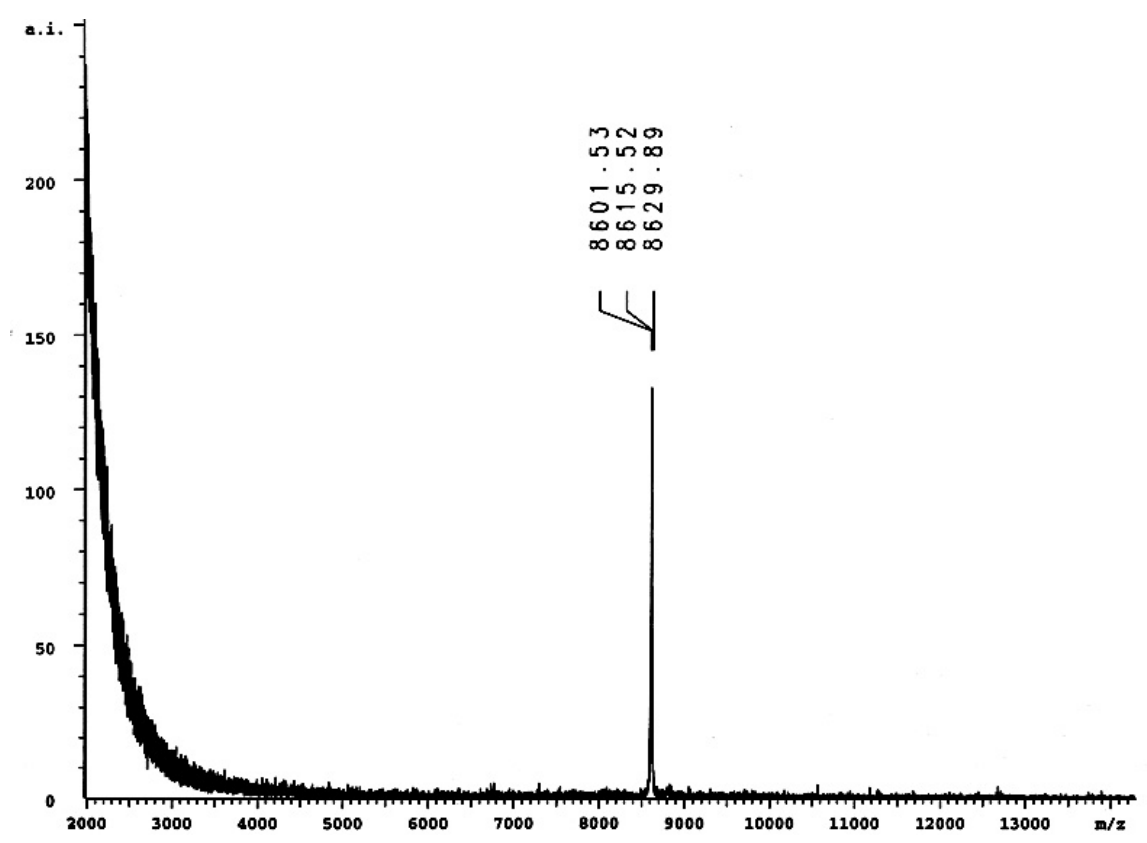

Figure S1: MALDI mass spectrum of TJ10 expressed in XL1-Blue E. coli. Expected mass: $8612.4 \mathrm{~g} / \mathrm{mol}$; found: $8615.5 \mathrm{~m} / \mathrm{z}$. 


\section{IgG binding of TJ10}

To confirm the IgG binding ability of TJ10, an assay that was first developed by Sloan and Hellinga, ${ }^{4,5}$ was used. The Q33C mutant of HTB1 was labeled with the environmentally sensitive dye, acrylodan, to form FHTB1. The analogous labeled mutant of the B1 portion of streptococcal protein $\mathrm{G}$ (GB1) has been shown to exhibit increased fluorescence at $480 \mathrm{~nm}$ upon binding to the Fc portion of human IgG. Even though this assay was developed to probe GB1 binding to the excised Fc portion of human IgG, experiments in our laboratory show that it is also valid in a system utilizing full human IgG and HTB1, the hyperthermophilic variant of B1 (data not shown). Since every $\mathrm{IgG}$ molecule contains two Fc regions, the stoichiometry of binding was adjusted accordingly.

To a preformed complex of whole human IgG $(1.75 \mu \mathrm{M}, 3.5 \mu \mathrm{M} \mathrm{Fc})$ and FHTB1 $(5 \mu \mathrm{M})$ in $20 \mathrm{mM}$ Tris- $\mathrm{HCl}$ at $\mathrm{pH}=7.4$, was added increasing amounts of either TJ10 or the parent HTB1. The decrease in fluorescence emission at $480 \mathrm{~nm}$ (excitation $=392 \mathrm{~nm}$ ) was monitored on a PTI fluorimeter (814 photomultiplier detection system and LPS-220B lamp power supply) as a function of increasing competitor concentration. This competition between FHTB1 and TJ10 for IgG binding demonstrates the ability of TJ10 to bind IgG.

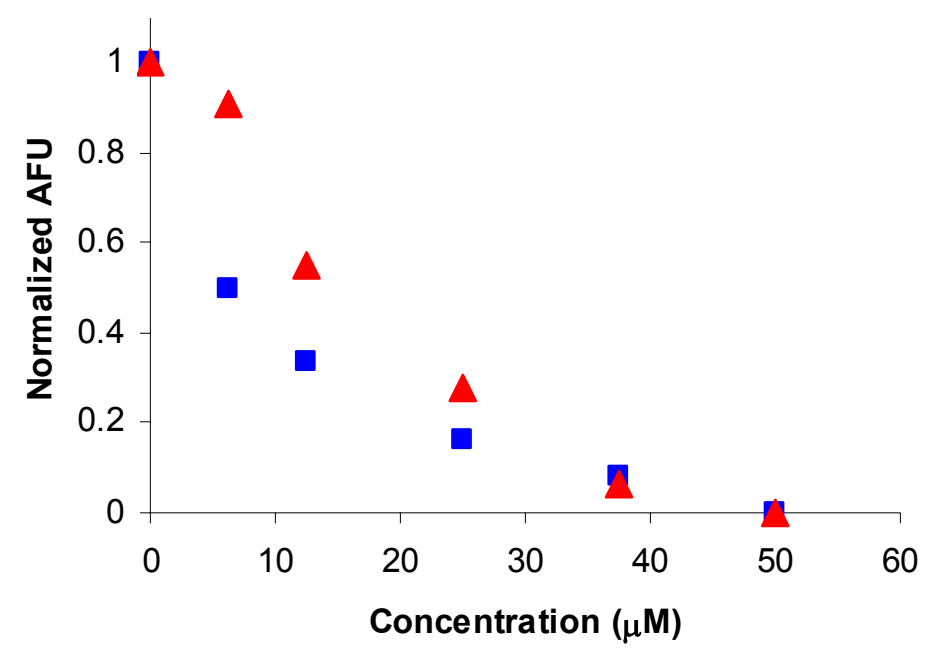

Figure S2: IgG binding of TJ10 through its helical surface opposite to the randomized beta-sheet surface. Increasing amounts of unlabeled HTB1 (a) and TJ10 ( $\boldsymbol{\Delta})$ were titrated into a complex of human IgG $(1.75 \mu \mathrm{M}, 3.5 \mu \mathrm{M} \mathrm{Fc})$ and FHTB1 $(5 \mu \mathrm{M})$. Fluorescence at $480 \mathrm{~nm}$ was normalized to the complex with no inhibitor. 


\section{Circular Dichroism (CD) and thermal stability of TJ10}

To determine if TJ10 retained a stable secondary structure, circular dichroism spectroscopy and a thermal denaturation were carried out. CD spectra were recorded on a JASCO J-710 spectropolarimeter in $20 \mathrm{mM}$ Tris- $\mathrm{HCl}(\mathrm{pH}=7.4)$ using a cuvette with a $0.1 \mathrm{~cm}$ pathlength. Mean residue elipticity was calculated as $(\theta \times 100) /(0.1 \mathrm{~cm} \times[\mathrm{P}] \times n)$, where $n$ is the number of residues, $[\mathrm{P}]$ is the protein concentration $(\mu \mathrm{M})$, and $\theta$ is raw elipticity. Thermal denaturation experiments were run in $3 \mathrm{M}$ guanidine- $\mathrm{HCl}$ and $20 \mathrm{mM}$ Tris- $\mathrm{HCl}(\mathrm{pH}=7.4)$ with a $2{ }^{\circ} \mathrm{C}$ step size while monitoring at $218 \mathrm{~nm}$ with an averaging time of $10 \mathrm{sec}$ and an equilibration time of $2 \mathrm{~min}$. A full spectrum and thermal melt are shown in the main manuscript. The observed secondary structure, even under the denaturing conditions of $3 \mathrm{M}$ guanidine- $\mathrm{HCl}$, demonstrates the retention of stable structure seen in the parent HTB1. ${ }^{1}$ The CD spectra (210$260 \mathrm{~nm}$ ) taken before and after the thermal melt show that it is a reversible process.

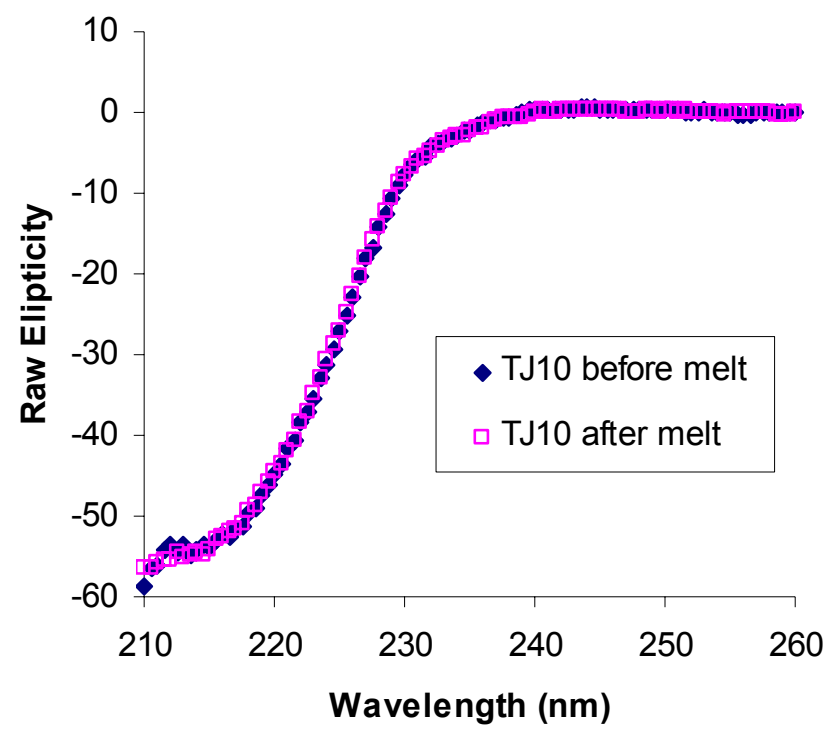

Figure S3: Circular dichroism spectra before and after a thermal melt. A thermal melt was carried out on $\mathrm{TJ} 10$ in $3 \mathrm{M}$ guanidine- $\mathrm{HCl}$ and $20 \mathrm{mM}$ Tris- $\mathrm{HCl}(\mathrm{pH}=7.4)$. CD spectra before and after the melt were recorded at $25^{\circ} \mathrm{C}$. 


\section{Determination of beta-amyloid fibril formation}

The formation of beta-amyloid fibrils was determined using the fluorescent histological dye, thioflavin T. Fibril formation was found to be very dependent upon the preparation of the beta-amyloid (A $\beta$ ) samples. $500 \mu \mathrm{g}$ of beta-amyloid (Sigma, Lot 59H49552) was mixed with $4.63 \mathrm{~mL}$ HFIP to yield $25 \mu \mathrm{M}(108 \mu \mathrm{g} / \mathrm{mL})$. The solution was sonicated in a bath sonicator for 2 hours to produce uniform beta-amyloid, and aliquoted samples were frozen at $-78{ }^{\circ} \mathrm{C}$ for later use in fibril formation assays.

Immediately before use, frozen beta-amyloid samples were lyophilized for 15-20 minutes to produce a fluffy white solid. The beta-amyloid samples were then dissolved in phosphate buffered saline (PBS) with $1 \mathrm{mM}$ EDTA and $0.05 \% \mathrm{NaN}_{3}$ at $\mathrm{pH}=7.4$. Fibril formation assays were carried out with a nominal beta-amyloid monomer concentration of $50 \mu \mathrm{M}$ with appropriate concentrations of the TJ10 protein. These solutions were shaken at $37{ }^{\circ} \mathrm{C}(250 \mathrm{rpm})$ with portions removed for time-based fibril formation determination.

The extent of fibril formation of each sample was determined by addition of $5 \mu \mathrm{L}$ of the beta-amyloid solution to $120 \mu \mathrm{L}$ of a thioflavin $\mathrm{T}$ solution $(50 \mathrm{mM}$ glycine- $\mathrm{NaOH}, \mathrm{pH}=8.5)$. The fluorescence of the thioflavin $\mathrm{T}$ was measured by monitoring emission at $482 \mathrm{~nm}$ with an excitation of $450 \mathrm{~nm}$ on a PTI fluorimeter (814 photomultiplier detection system and LPS-220B lamp power supply). These results show a concentration dependence of the inhibition of fibril formation by TJ10. Thus, sub-stoichiometric amounts of TJ10 allow for a modulated inhibition of beta-amyloid fibril formation.

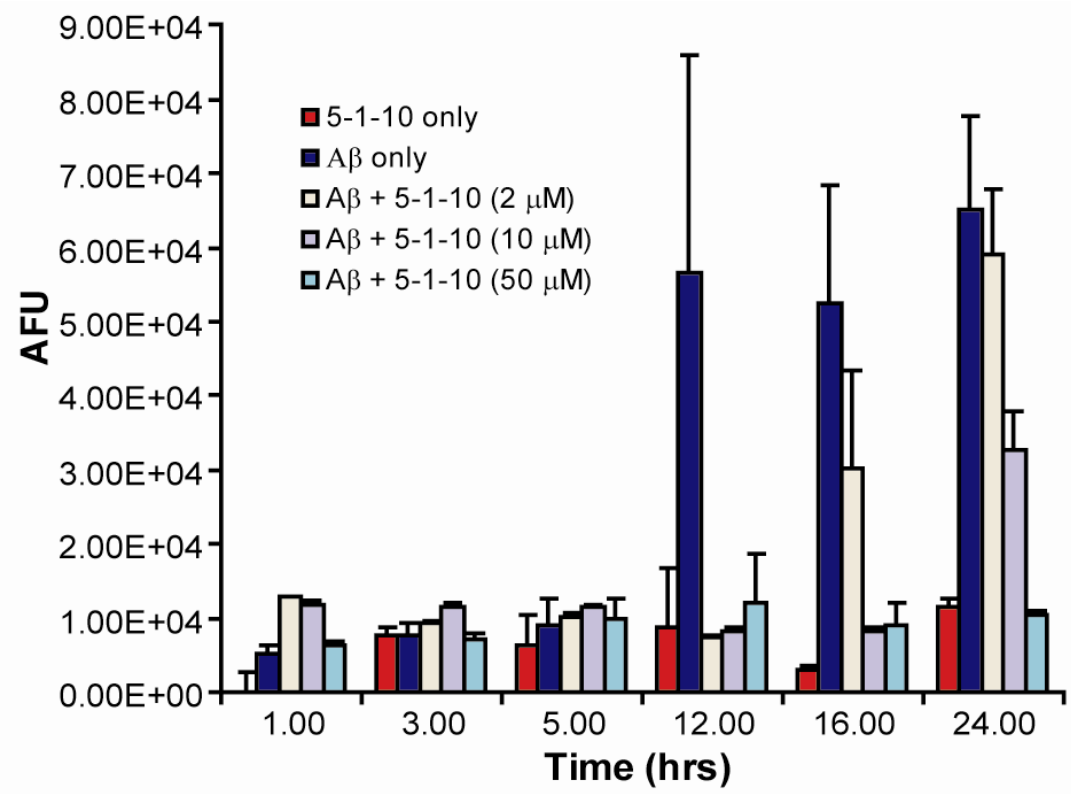

Figure S4: Concentration dependence of fibril formation inhibition. Beta-amyloid (A $\beta)$ at 50 $\mu \mathrm{M}$ nominal monomer concentration was mixed with TJ10 at $2 \mu \mathrm{M}, 10 \mu \mathrm{M}$, or $50 \mu \mathrm{M}$. The fibril formation was measured at various time points as the change in fluorescent emission of the histological dye thioflavin $\mathrm{T}$ at $482 \mathrm{~nm}$ with excitation at $450 \mathrm{~nm}$. 


\section{Effect of HTB1 on beta-amyloid fibril formation}

Multiple experiments were conducted to determine the effect of HTB1 on beta-amyloid fibril formation and a representative example is provided. Beta-amyloid $(25 \mu \mathrm{M})$ was mixed HTB1 $(54 \mu \mathrm{M})$ and fibril formation was monitored using the thioflavin T fluorescence assay described above, samples containing only beta-amyloid $(25 \mu \mathrm{M})$ or HTB1 $(54 \mu \mathrm{M})$ were also prepared. These experiments were repeated several times and a representative data set is shown below. These thioflavin $\mathrm{T}$ dependent fluorescence experiments clearly demonstrated that the parent HTB1 scaffold has no measurable effect on the aggregation properties of beta-amyloid (140).

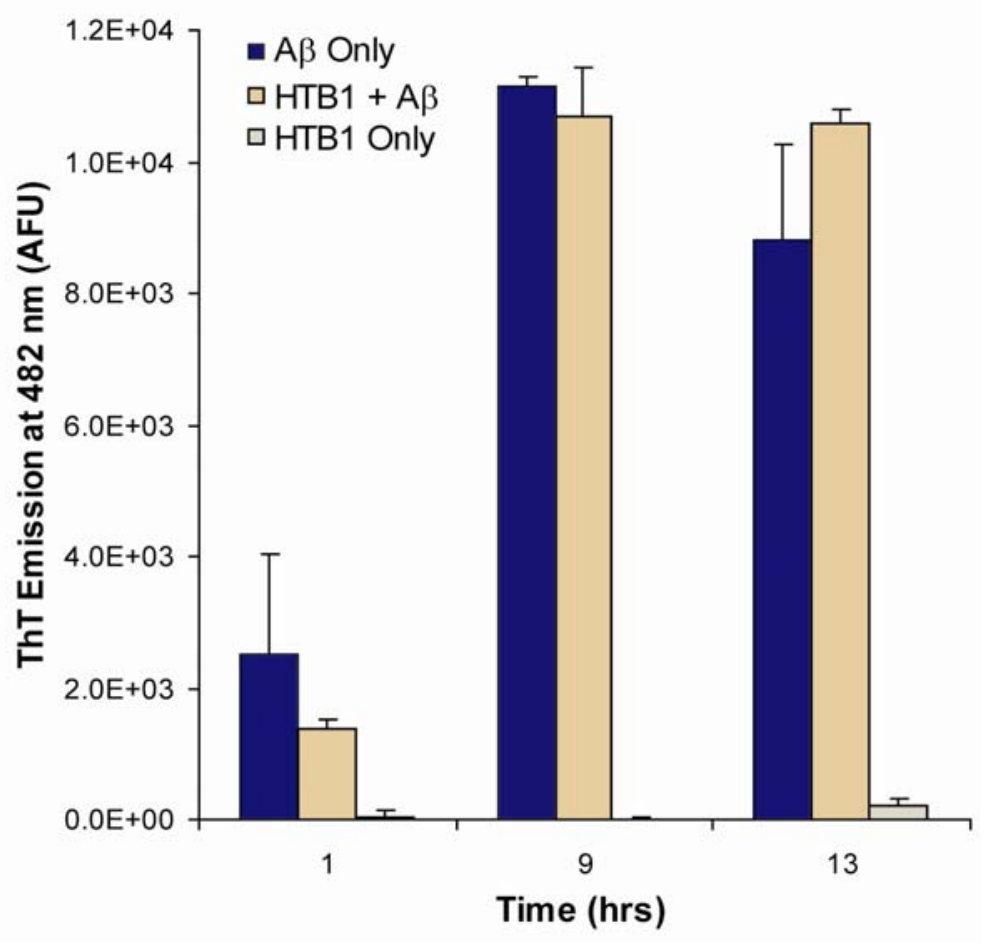

Figure S5: The effect of the parent scaffold, HTB1, on beta-amyloid fibril formation. Beta-amyloid $(25 \mu \mathrm{M})$ was mixed with HTB1 at $(54 \mu \mathrm{M})$. Fibril formation was monitored over time using thioflavin $\mathrm{T}$ dependent fluorescence as previously described.

\section{Transmission Electron Microscopy (TEM)}

Solutions of beta-amyloid with or without equimolar TJ10 were incubated (as described above) for two weeks. To each sample was added phosphotungstic acid ( $2 \%$ final, w/v), and the mixtures were placed onto the surface of a previously carbon-coated Piloform plastic (60-90 nm plastic, approximately $5 \mathrm{~nm}$ carbon) with a 150 mesh grid and were allowed to stand for 10 minutes. The excess buffer was withdrawn and the samples were allowed to dry. The samples were observed and photographed on a JEOL $100 \mathrm{CX}$ II TEM at $80 \mathrm{kV}$ with $40,000 \times$ magnification. An image from the sample without TJ10 is shown in the main text of the manuscript, but samples containing TJ10 showed no distinct fibril formation and the grids looked identical to those with only buffer. 


\section{Dynamic Light Scattering (DLS)}

Dynamic light scattering experiments were carried out on samples of beta-amyloid with or without the selected beta-amyloid-binding protein TJ10. Beta-amyloid $(25 \mu \mathrm{M})$ with or without TJ10 $(54 \mu \mathrm{M})$ was incubated for two weeks in phosphate buffered saline $(\mathrm{PBS}, \mathrm{pH}=$ 7.4) and $1 \mathrm{mM}$ EDTA at $37{ }^{\circ} \mathrm{C}$ with shaking at $250 \mathrm{rpm}$. Samples for light scattering determination were prepared by diluting $100 \mu \mathrm{L}$ of the fibril formation samples to $800 \mu \mathrm{L}$ in PBS. Light scattering data was collected at a $90^{\circ}$ angle on a BI8000 autocorrelator from Brookhaven Instrument Corp. ${ }^{6}$ Data was fit using a non-negatively constrained least squares method.

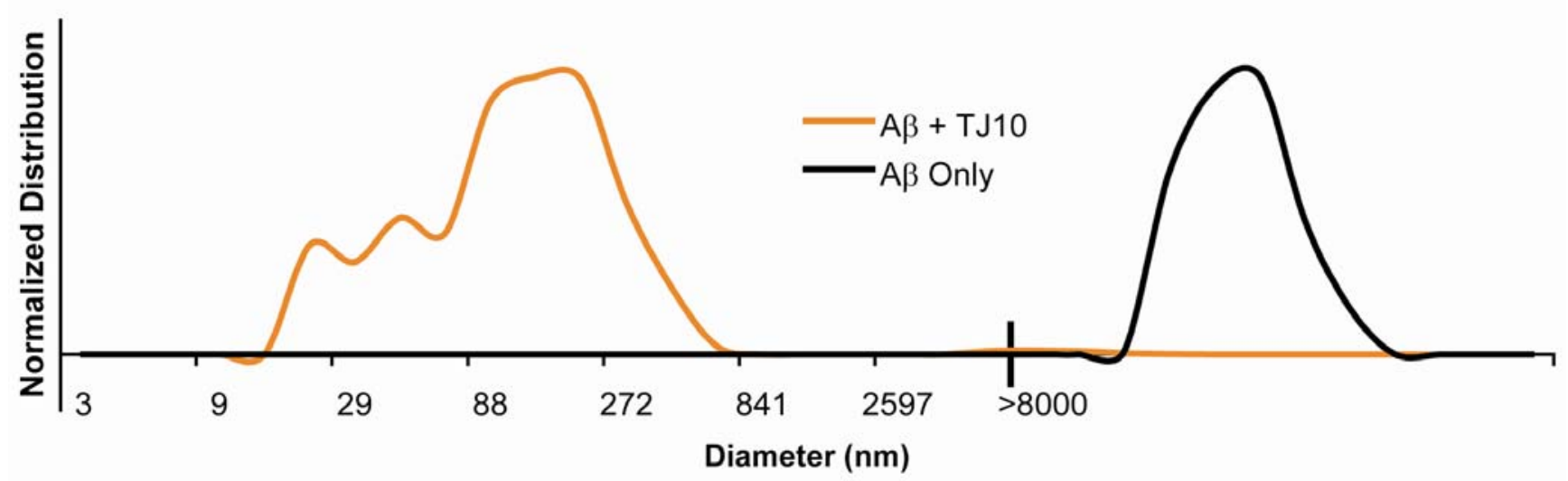

Figure S6: Dynamic light scattering of beta-amyloid. Light scattering experiments of beta-amyloid $(A \beta)$ samples were carried out after a two week incubation with or without TJ10.

\section{Tryptophan Fluorescence}

The tryptophan fluorescence of TJ10 was measured both in the presence and absence of pre-fibrillar beta-amyloid on a PTI fluorimeter (814 photomultiplier detection system and LPS220B lamp power supply). Fluorescent emission spectra were determined with excitation at both $270 \mathrm{~nm}$ and $290 \mathrm{~nm}$. TJ10 contains three tryptophan residues: two on the selected beta-sheet surface, and one from the parent HTB1. Beta-amyloid contains one tyrosine. A shift in the fluorescence spectrum upon beta-amyloid binding only occurs upon excitation at $270 \mathrm{~nm}$, indicating that there are multiple, distinct fluorescent species within TJ10, and that at the local environment of least one of these species is affected by beta-amyloid binding. 
Full Sequence of TJ10:

MRGSHHHHHHGSAAHYMAQTFWLSIQGKTLYWQIRIYAIDAAEAEKIFKQYANDNGIDGEWTYDDATKTFTVTE

Full Sequence of $A \beta$ :

DAEFRHDSGYEVHHQKLVFFAEDVGSNKGAI IGLMVGGVV

A)

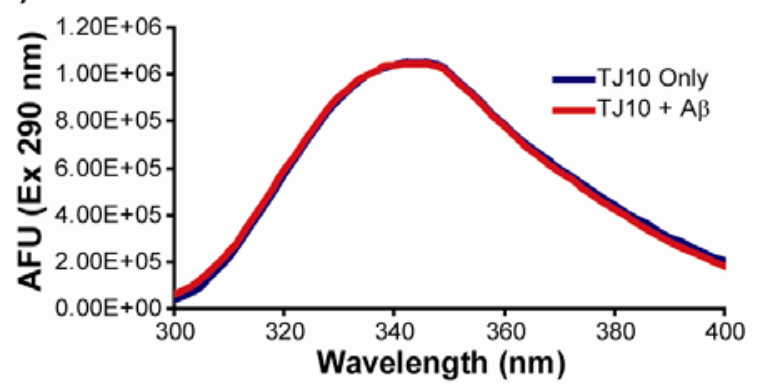

B)

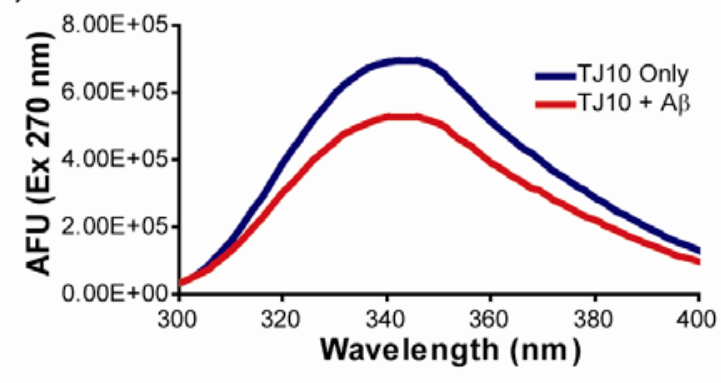

Figure S7: Tryptophan fluorescence emission spectrum with excitation at A) 290 $\mathrm{nm}$ and B) $270 \mathrm{~nm}$. Full sequences of each species, with Tyr in orange and Trp in red, are listed below for reference.

\section{Cited References}

(1) Malakauskas, S. M.; Mayo, S. L. Nat Struct Biol 1998, 5, 470-475.

(2) Meyer, S. C.; Huerta, C.; Ghosh, I. Biochemistry 2005, 44, 2360-2368.

(3) Chin, J. W.; Schepartz, A. Angew Chem Int Ed Engl 2001, 40, 3806-3809.

(4) Sloan, D. J.; Hellinga, H. W. Protein Sci 1999, 8, 1643-1648.

(5) Sloan, D. J.; Hellinga, H. W. Protein Eng 1998, 11, 819-823.

(6) Kolchens, S.; Ramaswami, V.; Birgenheier, J.; Nett, L.; O'Brien, D. F. Chem Phys Lipids 1993, 65, 1-10. 